\title{
Selection and Ordering of Number Entries in Four English Advanced Learner's Dictionaries
}

\author{
Yan Chen ${ }^{1}$ \\ ${ }^{1}$ Yancheng Teachers University, China \\ Correspondence: Yan Chen, Department of English Education, School of Foreign Languages, Yancheng Teachers \\ University, Yancheng 224002, Jiangsu, China. E-mail: cyan@xmu.edu.cn
}

Received: July 3, 2015 Accepted: July 27, 2015 Online Published: August 31, 2015

doi:10.5539/ells.v5n3p102 URL: http://dx.doi.org/10.5539/ells.v5n3p102

\begin{abstract}
This paper, by surveying the number entries in four English advanced learner's dictionaries, reveals some principles for their selection and ordering, as well as some problems to be reconsidered. It is found that besides the shift in grammatical function from numeral to other parts of speech, an overriding principle for inclusion is acquisition of new meaning, based on but extended or transferred from their cardinal or ordinal utility. Another principle, concerning numbers used in emails and text messages, seems to be neat phonetic correspondence between numbers and the words or word parts they stand for. To determine the eligibility for inclusion of the latter type of numbers, lexicographers may need to establish a frequency-formality criterion. The arrangement of number entries is typically represented by the ordering in LDOCE5 and that in CALD3. An experiment has shown that the ordering in LDOCE5 is more user-friendly.
\end{abstract}

Keywords: number entries, English advanced learner's dictionaries, selection, ordering

\section{Introduction}

Number is a "concept associated with quantity, size, measurement, etc., and represented by a word such as three, a symbol such as 3, a group of words such as eighty-three point five, or a group of symbols such as 83.5 " (McArthur, 1997, p. 410). In the world today, Arabic numerals, that is, the ten digits 0, 1, 2, 3, 4, 5, 6, 7, 8, and 9, are the most frequently used symbols to represent numbers. Numbers represented in this way, in fact, are employed not only as mathematical objects to count, label, and measure, but also as words to convey rich information. Hence the column "Numbers That are Entries" in Macmillan English Dictionary for Advanced Learners (hereafter $M E D A L$ ). In those entries, numbers containing one or more Arabic numerals are provided with pronunciation, grammatical information, definition, examples, and some even with style specifications, in precisely the same way a headword is treated. In other words, the numbers have acquired the properties of a word in every sense of the word and won a room in dictionaries. World-renowned English advanced learner's dictionaries (hereafter ALDs), including Oxford Advanced Learner's Dictionary (hereafter OLAD), Longman Dictionary of Contemporary English for Advanced Learners (hereafter LDOCE) and Cambridge Advanced Learner's Dictionary (hereafter $C A L D$ ), have all devoted some space to the treatment of numbers, at least in their latest editions. None of those dictionaries, regretfully, have offered any guidelines on the inclusion or treatment of those numbers. Meanwhile, no monographs or essays on lexicography have dealt with any theoretical issues concerning their treatment. However, with the increasing popularity of numbers used as words in daily life, dictionary makers, as faithful recorders of language change, are obliged to probe into the phenomenon both in practice and in theory. In this paper, I will attempt to induce some principles for their treatment through a thorough examination of entries available, particularly principles for selection and ordering of numbers. For the sake of convenience, the entries dealing with numbers will be referred to as number entries.

\section{A Survey of Number Entries in Four ALDs}

\subsection{Clarification of Number Entries}

In the above-mentioned four dictionaries, numbers are treated in both the front matter and the back matter. In the front matter, numbers are treated in the "Numbers (and symbols)" column right before the dictionary body, as in OALD8 (2010) and LDOCE5 (2009), or in the "Numbers That are Entries" or "Numbers that are used as words" column as in MEDEL2 (2007) and CALD3 (2008). In the back matter, by contrast, the numbers section may 
come as a single column, as in $O A L D 8$, or integrated into a section on "Numbers, weights and measures", as in LDOCE5. Numbers in the front matter differ sharply from those in the back matter. As is mentioned above, the former are presented in a dictionary entry format, whereas the relevant back matter sections explain how to write and say numbers, what numbers represent and so on, just as numbers, weights and measures are traditionally presented in dictionaries. This leads to the conclusion that numbers in the front matter are treated as lexical items while those in the back as mathematic objects.

The difference is further clarified by the column titles and subsequent explanations. According to explanations in CALD3, "[y]ou will sometimes find these numbers used like ordinary words in English, especially in newspapers or on the Internet. This page tells you what they mean and how they are used." The wording indicates that lexicographers implicitly agree that the numbers are similar to words in function and usage and deserve to be treated like words. However, due to the foreign nature of Arabic numerals and their apparent incompatibility with the English alphabet, or probably their small number as against the large number of English headwords, the numbers cannot fit readily into the usual alphabetical order of the headwords in the dictionary body. Consequently, they are set off from the dictionary body as independent columns, without so much as a uniform designation for the numbers section. Anyway, "number entries" may serve as an expedient name that covers their nature and form of presentation.

It is an undeniable fact that number entries are drawing more and more attention from lexicographers. LDOCE4 (2003), for example, contains no number entries, but LDOCE5 has incorporated 24 such entries. The OALD series has increased the number of number entries from 16 in OALD6 (2000) to 18 in OALD7 (2005) and 24 in $O A L D 8$, and the MEDAL series from 37 in MEDAL1 (2002) to 40 in MEDAL2. It is high time to put number entries into perspective.

\subsection{Semantic and Grammatical Features of Numbers in Number Entries}

The numbers covered in number entries fall into two types: pure Arabic numerals such as " 2.1 " and " $24 / 7$ ", and a combination of Arabic numerals and English forms like " $3 \mathrm{G}$ " and " 18 -wheeler". It should be noted that the numbers in question refer to Arabic numerals representing amounts or quantities, excluding English words for numbers as in common usage. An entry-by-entry examination of number entries has revealed the following semantic and grammatical features of numbers included in the entries.

First and foremost, the number not only fulfils the function of a cardinal or ordinal number for counting, measuring, labeling, and ordering; it has a new and lexical meaning of its own as well, which is manifested in several ways.

The first manifestation is that some numbers have changed from mathematic objects into proper names to identify certain referents. The number " $9 / 11$ ", for instance, is not just a date; it is "the abbreviation for the date September 11, 2001, when terrorists flew planes into the World Trade Center in New York, the Pentagon in Washington, D.C., and a field in Pennsylvania, killing thousands of people" (OALD8). Similarly, the number " $7 / 7$ " is "used to refer to July 7, 2005, when four suicide bombers killed themselves and 52 other people in attacks on London's public transport system" (CALD3).

The second manifestation is that some numbers have become special symbols used to refer to objects of a special kind. In other words, their grammatical function has shifted from numeral to common noun. The numbers " 12 ", " $15 "$, " 18 ", for instance, are used to mark films that cannot be legally watched by children under a specific age, as in I can take the kids too - it's a 12. The numbers ".22" and ".45" have become countable nouns denoting guns that take bullets which are .22 or .45 inches in diameter respectively (LDOCE5). In the same way, "99" is "an ice cream in a cone with a stick of chocolate in the top", and " $4 \times 4$ " is "a vehicle with a system in which power is applied to all four wheels, making it easier to control" (OALD8).

The third manifestation is that some numbers have undergone various changes of meaning. One change is generalization. The number "24/7", literally "twenty-four hours a day, seven days a week", is extended to mean "all the time" (MEDAL2). The same change is also seen in "411", the telephone number of the service used in the US to find out a person's telephone number, which in informal usage means "the true facts about a situation or the information you need", as in He'll give us the 411 on what to expect (OALD8). "The \$64,000 question", "[f]rom a popular US TV game show in the 1950s called The $\$ 64,000$ Question on which people could win up to $\$ 64,000$ by answering more and more questions correctly," now refers to "a question that is the most important and most difficult to answer concerning a particular problem or situation" (MEDAL2). Another change is transference of meaning, as is seen in "20/20 vision", which is used metonymically to mean the ability to see perfectly without using glasses or contact lenses (OALD8). A similar case in point is "404", which originates from the usual message which appears on the Internet when a particular page one is searching for cannot be 
found, but has turned into a humourous adjective to describe someone who is "stupid and unable to use computers or other complicated electronic equipment" (CALD3).

Furthermore, in terms of grammar, the number in most number entries has changed into a different grammatical category, the most common type being from numeral to noun. In $O A L D 8,19$ out of the 24 numbers are labeled as noun, and in LDOCE5, 14 out of 24. A less common type is from numeral to adjective, like "404", "3-D", " $3 \mathrm{G}$ ", and " $101 "$. A rare case is the number " 86 ", which has turned into a verb, meaning "to get rid of someone or something that is not wanted" (MEDAL1). Equally rare is " $24 / 7$ ", which may function either as an adverb or as an adjective (CALD3).

\subsection{Ordering of Numbers in Number Entries}

In number entries, the numbers are found to be arranged in varied orders. In $L D O C E 5$, the numbers are ordered from the smallest to the largest, in ascending order. Special symbols (such as "\$”, "x", "." and " "”) do not count. So the list begins with the smallest value ".22", keeps going up to 3 (as in " $3 G$ "), 24 (as in " $24 \times 7$ "), 401 (as in " $401 \mathrm{k}$ "), etc., and ends up with the largest value 64,000 (as in "the \$ 64,000 question"). In the same way, 20 (as in "20/20 vision") sits between 18 (as in "18-wheeler") and 24 (as in "24-7"). If the first numeral for two numbers is equal to each other, for example, " $4 \mathrm{x} 4$ " and " $4-\mathrm{H}$ " both having 4 , then the second alphanumeric decides their ranking, with numeral ranking before alphabet. Therefore, " $4 \times 4$ " precedes " $4-\mathrm{H}$ ".

In $C A L D 3$, by contrast, numbers are arranged in different groupings, with initial numeral in ascending order from 0 to 9, regardless of symbols like the decimal point “.” and the dollar sign " $\$$ ". All numbers beginning with 0 are arranged in the first grouping with initial numeral 0 . All numbers beginning with 1 are arranged in the second grouping with initial numeral 1, etc. All numbers beginning with 9 are arranged in the last grouping with initial numeral 9. Within each grouping, numbers are sorted in ascending order alphanumerically. Numbers are listed from small to large values; alphabets from A to Z. Values are compared from the left to the right, with numeral ranking before alphabet. For example, "0800 number" and "0898 number", both beginning with 0 , are put into the beginning group, sorted in ascending order, and then followed by numbers beginning with 1 , for example, "101", "12A", "15", "18", "180" sorted in ascending order too. "24/7" precedes "24-hour clock", and " $3 \mathrm{G}$ " goes before " $3 \mathrm{Ws".} \mathrm{Such} \mathrm{an} \mathrm{ordering} \mathrm{system} \mathrm{is} \mathrm{also} \mathrm{found} \mathrm{in} \mathrm{MEDAL1,} \mathrm{with} \mathrm{the} \mathrm{addition} \mathrm{of} \mathrm{a} \mathrm{minor}$ ordering principle: in numbers beginning with the same numerals, symbols like "/" ranks before numerals, as in the precedence of " $9 / 11$ " over " 911 ".

In $O A L D 8$, which follows basically the same ordering principle as $C A L D 3$, some complexities are detected. First, the ascending order of numbers is broken when "the $\$ 64,000$ questions", which is supposed to go between " 411 " and " $7 / 7$ ", actually sits between " $7 / 7$ " and " $9 / 11$ ", thus forming an inconsistency. Secondly, the precedence of "24-hour clock" over " $24 / 7$ " suggests a change in the ordering of numbers beginning with the same numerals: alphabet ranks before rather than after numeral, obviously a reversal of the ordering in LDOCE5 and CALD3. In addition, $O A L D 8$ follows the minor principle adopted in $M E D A L 1$ : precedence of symbols over numerals in numbers beginning with the same numerals.

\section{Problems to Be Reconsidered}

\subsection{Selective Principles and Restrictions Regarding Numbers}

"In any case, the selection of the entries for the dictionary is a highly delicate task" (Zgusta, 1977, p. 248). The selection of numbers, undoubtedly, must be made on principles applied to dictionaries in general, including authenticity, representativeness, coverage, suitability, etc. (Svensén, 1993, pp. 40-53). From the above survey, it may be safely stated that the numbers included have exhibited features conforming to those of a word on phonetic-phonological level, orthographic-graphemic level, morphological level, lexical-semantic level, and syntactic level (cf. Bussmann, 2000, p. 521). The use of those numbers may have been so stabilized and widespread that they constitute a special category of linguistic units worthy to be included and equipped with a great range of semantic, grammatical and stylistic information. In addition to the shift of grammatical function from numeral to proper or common noun and other parts of speech, an overriding principle for inclusion of numbers to be treated in entries, as shown in the above survey, is the acquisition of new meaning, based on but extended or transferred from their cardinal or ordinal utility.

Another principle for selection of numbers concerns a different type of numbers hardly touched upon in most ALDs. In MEDAL series alone, four natural numbers are included in number entries, that is, 1, 2, 4 and 8, all of which are abbreviations "used in emails and text messages" to replace "-one", "to" or "too", "for" or "-fore", and "-ate" or "-eat", respectively, as in "NE1(=anyone)", "2day (=today)", "me 2 (=me too)", "4 U (=for you)", "B4 (=before)", "GR8 (=great)" and "L8R (=later)". Similar information is found in the back matter of LDOCE5, 
where a subsection under the numbers section deals with the use of 1, 2, 4 and 8 in text messages. These numbers, according to LDOCE5, are ways of "writing" words (that is, one, to, too, for) or part of words as in the use of "8" to "write" word parts that sound like "-ate", "eat", or "-ait", like "gr8 (=great)", "h8 (=hate)", and "18 (=late)". As part of chat-room and texting language, these numbers have lost their functions as cardinal or ordinal numbers; they are nothing more than homophonic numerical transcriptions of words or word parts, hence my coinage pseudo-numbers. Then a question arises: What are the criteria for their inclusion? A principle seems to hold up well if a comparison is made between these numbers and the words or word parts they stand for: neat phonetic correspondence between them. The very correspondence not only makes the use of those numbers predictable and readily intelligible, but also contributes to avoiding ambiguity or misunderstanding in communication. Thanks to such correspondence, it is possible for lexicographers to provide a summary indication of the instances where these numbers occur, and rather exhaustively.

The use of pseudo-numbers in daily life is nothing new, due to the prevalence of the Internet and mobile phone. Apart from the above-mentioned examples, a lot more pseudo-numbers are in current use, on Twitter, Facebook and elsewhere, "121 (=one to one)", "2morow (=tomorrow)", "2nite (=tonight)", "2U2 (= to you too)", "M8 (=mate)", to cite just a few. The number of numbers used in this way in the general language has kept on increasing, and the trend will continue. A check at http://www.chatslang.com/terms/online chat will show that some pseudo-numbers do not correspond neatly to what they represent in pronunciation, like "10Q (=Thank you)", "10x (=Thanks)", "3rzda (=Thursday)", "4Q (=fuck you)", "h4x0r (=hacker)", "I8U (=I hate you)", "n8kd (=naked)", "f9 (=fine)". Meanwhile, the usage of numbers has gone far beyond phonetic representation of words. According to the Urban Dictionary, the number "143" is a numerical representation of the expression "I love you" where each of the three numbers represents the quantity of letters in each of the words, and " 459 " means "I love you" on a North American telephone keypad. Under such circumstances, indiscriminate inclusion of such diverse and novel usages of numbers will be promiscuous and haphazard; restrictions need to be set as regards their eligibility for inclusion.

As the numbers used in chat-rooms, emails and text messages are mostly everyday informal language, they may have high frequency but may be short-lived. Lexicographers cannot easily and unequivocally decide which numbers to include and which to exclude, but neither can they wait until they have strong evidence to prove if a number has stood the test of time to be worthy of inclusion. "One of the principal selling points of dictionary publishers is that the new dictionary contains as much as possible of "the very latest material" (Svensén, 1993, p. 47). MEDAL1 has broken new ground in the treatment of pseudo-numbers, but the practice of the other three ALDs is rather conservative. In my opinion, a dictionary revised as often as $O A L D$ can afford to include pseudo-numbers more generously, to increase the descriptive and informative power of the dictionary. What is needed, I think, is to establish a frequency-formality criterion for lexicographers to determine the eligibility of pseudo-numbers for selection. The availability of text corpora, databases, and sophisticated software programs allow lexicographers to undertake researches into the frequency of occurrence of pseudo-numbers, so that they may distinguish popular and uniform usages from rare and ephemeral ones. The level of formality is an equally important factor in the selective decision, due to the great prescriptive power of dictionaries. Offensive usages of numbers and numbers expressing questionable value-judgments, for example, "4Q", "53x (=sex)", "CU46 (=See you for sex)", should be excluded so as to reduce the risk of raising them to the status of normal or accepted usage.

\subsection{More User-Friendly Ordering of Numbers}

As is shown in my survey, there are no uniform norms for the ordering of numbers in ALDs. To test the effectiveness of different ordering systems, I conducted an experiment among two classes of Chinese undergraduate English majors, one class of 31 freshmen and the other of 40 sophomores. When asked to locate a specific number, like "24/7" and "999" in LDOCE5 or CALD3, the learners had no difficulty at all. This is understandable as there is only one page of such numbers in each dictionary and it took them only a short time to skim through the whole list. When asked to identify the patterns of ordering of numbers, the same learners reacted very differently. It took them about two minutes to identify the "smallest to largest value" arrangement of numbers in $L D O C E 5$, but the same students could barely discern any patterns of ordering in CALD3. After I explained how the numbers are ordered, they expressed a strong dislike for the ordering, because they are used to counting from single digits to double digits, triple digits, and so on, and because the ordering from " 0800 number" to "0898 number", "101", "12A", "15", "18", "180", etc., is not in keeping with their intuition to compare the value of integers first through their length. For users of $C A L D 3$, the consultation process consists of more than two steps: finding the section regarding a specific initial numeral, and comparing the values of the second or third or more numerals from left to right. More time and effort in consultation is an obvious 
impediment to the user-friendliness of the work.

Despite the fact that the CALD3 ordering of numbers is also found in MEDAL and OALD and that the LDOCE5 ordering is an individual case, future lexicographers need to reconsider the strengths and weaknesses of both ordering systems. The above simple findings may be of some value when the list of numbers gets longer and longer as more and more numbers are included.

\section{Conclusion}

The number of numbers treated as words in ALDs, so far, is quite small, but this does not undermine their importance as a particular subset of the total lexicon of English. Semantically, numbers in number entries have acquired new and lexical meanings of their own in addition to fulfilling the function of cardinal or ordinal numbers for counting, measuring, labeling, and ordering. Grammatically, the numbers in most number entries have changed from numeral into other grammatical categories like noun, adjective, verb, adverb or adjective. My examination of four ALDs has revealed that acquisition of new meaning and change of part of speech are a dual criterion for the selection of numbers in number entries and that for numbers in chat-room and texting language, neat phonetic correspondence between numbers and the words or word parts they stand for is a must for inclusion. So far, no uniform norms have been established for the ordering of numbers in ALDs, so it remains to be explored what a truly user-friendly arrangement of number entries is. To fulfill learners' receptive and productive needs, ALD makers need to be comprehensive, balanced and user-friendly in the coverage and treatment of numbers. My discussion is but a shallow observation open to doubt as there are no norms generally agreed upon in the treatment of numbers. It is my hope that future editions of ALDs will perfect the presentation of numbers.

\section{References}

Bussmann, H. (Ed.). (1996). Routledge dictionary of language and linguistics. London: Routledge.

Hornby, A. S. (Ed.). (2010). Oxford advanced learner's dictionary (8th ed.). Oxford: Oxford University Press. $(O A L D 8)$

Mayor, M. (Ed.). (2009). Longman dictionary of contemporary English for advanced learners (5th ed.). London: Pearson Education Limited. (LDOCE5)

McArthur, T. (Ed.). (1997). The concise companion to the English language. Oxford: Oxford University Press.

Rundell, M. (Ed.). (2007). Macmillan English dictionary for advanced learners (2nd ed.). London: Macmillan. (MEDAL2)

Svensén, B. (1993). Practical lexicography: Principles and methods of dictionary-making. Oxford: Oxford University Press.

Walter, E. (Ed.). (2008). Cambridge advanced learner's dictionary (3rd ed.). Cambridge: Cambridge University Press. (CALD3)

Zgusta, L. (1971). Manual of lexicography. The Hague: Mouton. http://dx.doi.org/10.1515/9783111349183

\section{Copyrights}

Copyright for this article is retained by the author(s), with first publication rights granted to the journal.

This is an open-access article distributed under the terms and conditions of the Creative Commons Attribution license (http://creativecommons.org/licenses/by/3.0/). 